\title{
Noise propagation in a class of metabolic networks
}

\author{
A. Borri P. Palumbo A. Singh
}

\begin{abstract}
A metabolic pathway made of a cascade of biochemical reactions is considered, with a substrate which is eventually transformed into the final product by means of a sequence of reactions, each catalyzed by the same enzyme. The amount of the enzyme varies according to discrete noisy processes of production and elimination. A feedback acts on the final product clearance rate, exerted by the final product accumulation itself: higher final product levels lead to a faster dynamics. The aim of this note is to investigate how the noise scales with the length of the cascade and how the feedback impacts on the noise propagation. To this end, a Stochastic Hybrid System (SHS) formulation is exploited, with the enzyme production/clearance processes constituting the noise source. The noise propagation is measured in terms of the square of the coefficient of variation of the final product, and computations are carried out by means of the equations of moments, which are estimated in closed form after linearizing the SHS. Analytical solutions allow to infer information and to relate the noise propagation to the model parameters. Similarly to recent results occurring in other types of enzymatic reactions, the results highlight the influential role of feedback in noise reduction.
\end{abstract}

Index Terms-Metabolic pathways, Moment Equations, Negative Feedback

\section{INTRODUCTION}

In the last few years, mathematical control theory has been increasingly applied in Systems and Synthetic Biology. On the one hand, the aim of Systems Biology is to understand, quantify and conceptualize the diverse frameworks involving complex biological systems, possibly taking inspiration from established engineering paradigms: one may cite, among the others, the role of feedback, which has been widely investigated, especially in transcriptional and metabolic regulation where gene products are required to control their homeostatic levels robustly with respect to parameter or environmental fluctuations [1], [2], [3], [4], [5], [6], [7], [8], [9], [10], [11]; on the other hand, Synthetic Biology aims at merging molecular biological techniques with mathematical modeling and forward engineering in order to design synthetic biological circuits, able to replicate emergent properties potentially useful for biotechnology industry, human health and environment (see [12], [13], [14], [15], [16] and references therein).

This note considers the case of a metabolic pathway, with a substrate $p_{0}$ undergoing a cascade of $n$ metabolic modifications, leading to the final product $p_{n}$. Substrate

A. Borri, and P. Palumbo are with the Istituto di Analisi dei Sistemi e Informatica "A. Ruberti", Italian National Research Council (IASI-CNR), Via dei Taurini, Roma, Italy. Email addresses: \{alessandro.borri, pasquale.palumbo\}eiasi.cnr.it

A. Singh is with Department of Electrical and Computer Engineering, Biomedical Engineering, Mathematical Sciences, Center for Bioinformatics and Computational Biology, University of Delaware, Newark, DE USA 19716. E-Mail address: absingh@udel . edu production and final product clearance are also accounted for, see the scheme in Fig. 1. Each substrate modification is catalyzed by enzymes: the assumption is that there exists only one enzyme acting on all the biochemical reactions. The amount of the enzyme varies according to discrete noisy processes of production and elimination. One may think at the proposed scheme as to a simplified paradigm for a wide range of sequential events like, e.g. cascades of phosphorylations. The final product accumulation acts in feedback to speed up its own dynamics. This fact has been recently investigated in [17] at a single-cell level, where an apparent correlation between the metabolic level and the growth rate has been highlighted, while a similar cascade scheme, related to gene expression, was investigated in a different fashion in [18].

The aim of this note is to investigate how the noise scales with the length $n$ of the cascade and how the feedback impacts on the noise propagation. To this end a Stochastic Hybrid System (SHS) is exploited, with the enzyme updates (production and clearance) being the only noise sources of the system. Noise is measured in terms of the squared coefficient of variation of the final product. Computations are carried out by means of the moment equations [19], [20], [21]: because of the nonlinearities involved by the Ordinary Differential Equation (ODE) system associated to the SHS, computations are made after linearization, so that the moment equations come out in closed form. Despite the simplifications adopted, an explicit easy-to-handle solution is not provided, in favor of a recursive algorithm releasing the second-order moment associated to $p_{n}$. Results coming after linearization are validated by the Gillespie Stochastic Simulation Algorithm (SSA), run according to the original nonlinear system, which is shown to be very close to the approximated one on a wide range of model parameters.

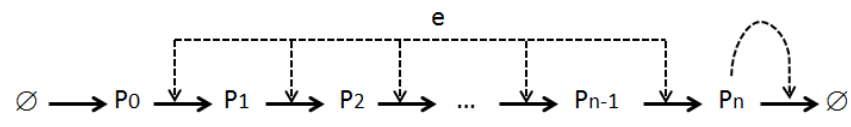

Fig. 1. Cascade of biochemical reactions.

Similarly to recent results occurring in other types of enzymatic reactions [7], [9], [10], [8], results highlight the influential role of the feedback in noise reduction.

The note is organized as follows. Section 2 is devoted to introduce the SHS mathematical model describing the metabolic pathway. First and second order moments are computed in Sections 3 and 4, showing how they are related to the model and feedback parameters. Section 3 is devoted 
TABLE I

CHEMICAL REACTIONS.

\begin{tabular}{|c|c|c|}
\hline Event & Enzyme reset & Propensity function \\
\hline Enzyme production & $e(t) \mapsto e(t)+1$ & $a_{1}=k_{e}$ \\
\hline Enzyme clearance & $e(t) \mapsto e(t)-1$ & $a_{2}=\gamma_{e} e(t)$ \\
\hline
\end{tabular}

to introducing the linear approximation as well, which is achieved in the neighborhood of the steady-state of the system. Section 5 reports simulation results carried out in order to stress the improvements in noise reduction achieved by the feedback.

\section{METABOLIC PATHWAY MODEL}

Consider the scheme in Fig. 1, where a substrate is sequentially modified $n$ times passing from state 0 , to state 1 and, eventually, to state $n$. These modifications are supposed irreversible. Denote with $p_{i}, i=0,1, \ldots, n$ the copy number of the substrate in state $i$. By assuming standard massaction law to describe the substrate modifications catalyzed by a unique enzyme (whose copy number is denoted by $e$ ), the ODE system associated to the metabolic pathway under investigation is:

$$
\begin{aligned}
\frac{d p_{0}}{d t} & =k_{p}-\frac{\lambda}{e^{\star}} e(t) p_{0}(t) \\
\frac{d p_{i}}{d t} & =\frac{\lambda}{e^{\star}} e(t) p_{i-1}(t)-\frac{\lambda}{e^{\star}} e(t) p_{i}(t) \quad i=1, \ldots, n-1 \\
\frac{d p_{n}}{d t} & =\frac{\lambda}{e^{\star}} e(t) p_{n-1}(t)-\gamma_{p} p_{n}(t)
\end{aligned}
$$

where $k_{p}$ stands for the $p_{0}$ substrate production rate, $\lambda$ is the rate of transformation from $p_{i}$ into $p_{i+1}$ (and is normalized by the average steady-state enzyme copy number, denoted by $\left.e^{\star}\right)$ and $\gamma_{p}$ stands for the final product clearance rate.

Motivated by [17], where an apparent correlation between the metabolic level and the growth rate has been highlighted, we introduce a feedback action exerted by the final product accumulation on the clearance rate $\gamma_{p}$, according to the following Hill function:

$$
\gamma_{p}\left(p_{n}\right)=\bar{\gamma}_{p} \frac{p_{n}^{h}}{\theta^{h}+p_{n}^{h}},
$$

where $\bar{\gamma}_{p}$ is the clearance strength, providing the maximal clearance rate, obtainable for negligible values of the repression threshold $\theta$ (negligible w.r.t. the $p_{n}$ copy number), $\theta$ indicates half of the maximal value reached for $p_{n}=\theta$, and parameter $h$ is the feedback sensitivity, providing the steepness of the sigmoidal function.

The copy number of the enzyme acting at catalyzing the substrate transformation is allowed to vary according to discrete noisy processes of production and clearance, whose related chemical reactions are summarized in Table I, detailing the resets on the enzyme and the propensities associated to both production/clearance processes.

In the following, unless differently specified, the expected value of a random variable $x$ is denoted by $\langle x\rangle$, while the steady-state of the average of a stochastic process $x(t)$ and of a second-order moment $\langle x(t) y(t)\rangle$ are denoted by $x^{\star}=\lim _{t \mapsto+\infty}\langle x(t)\rangle$ and $\langle x y\rangle^{\star}=\lim _{t \mapsto+\infty}\langle x(t) y(t)\rangle$, respectively.

\section{A. Metabolic noise computation}

In order to quantify how the noise scales with respect to the length of the metabolic pathway and the feedback action, we define the metabolic noise associated to the final product $p_{n}$ by means of the square of the coefficient of variation $C V_{p, n}^{2}$, computed by the ratio:

$$
C V_{p, n}^{2}=\sigma_{n}^{2} /\left(p_{n}^{\star}\right)^{2},
$$

where $\sigma_{n}^{2}$ and $p_{n}^{\star}$ are the steady-state values for variance and mean of the marginal distribution of the final product $p_{n}$ copy number. It readily comes out that, by varying the model or the feedback parameters, $C V_{p, n}^{2}$ may vary because of both the $p_{n}$ steady-state and the fluctuations around $p_{n}^{\star}$. Moreover, in order to compare the feedback and the no-feedback cases, the feedback relative noise is defined as the ratio

$$
\eta_{n}^{2}=\frac{C V_{p, n}^{2}(\text { feedback })}{C V_{p, n}^{2}(\text { no feedback })}
$$

where the no-feedback case refers to $\gamma_{p}\left(p_{n}\right)=\bar{\gamma}_{p}$, a situation occurring whenever the final product copy number is much larger than the threshold: $p_{n} \gg \theta$.

\section{AVERAGE STEADY-STATE SOLUTIONS}

The first-order moment equations associated to the SHS modeling the reaction schemes are derived from [19]. Since the enzyme is not influenced by the substrate, any order moment of $e$ can be computed without any approximation, readily providing the following results for the equilibrium first- and second-order moments:

$$
e^{\star}=\frac{k_{e}}{\gamma_{e}}, \quad\left\langle e^{2}\right\rangle^{\star}=\left(e^{\star}\right)^{2}+e^{\star},
$$

from which it comes that

$$
C V_{e}^{2}=\frac{\left\langle e^{2}\right\rangle^{\star}-\left(e^{\star}\right)^{2}}{\left(e^{\star}\right)^{2}}=\frac{1}{e^{\star}}=\frac{\gamma_{e}}{k_{e}} .
$$

As expected, the noise source becomes larger by reducing the average enzyme copy number.

On the other hand, the first-order moment equations applied to the SHS provide the following identities

$$
\begin{aligned}
& \left\langle e p_{0}\right\rangle^{\star}=\left\langle e p_{1}\right\rangle^{\star}=\cdots=\left\langle e p_{n-1}\right\rangle^{\star}=\frac{k_{p} e^{\star}}{\lambda} \\
& \left\langle\gamma_{p}\left(p_{n}\right) p_{n}\right\rangle^{\star}=\frac{\lambda}{e^{\star}}\left\langle e p_{n-1}\right\rangle^{\star}=k_{p}
\end{aligned}
$$

which do not allow any closed-form solutions because of the nonlinearities involved in the ODE associated to the SHS. To cope with this drawback, we linearize (around the steady states $e^{\star}$ and $p_{n}^{\star}$ ) the nonlinear function $\gamma_{p}\left(p_{n}\right) p_{n}$ as well as the mass-action law related to the intermediate substrate transformations, so that the ODE system associated to the 
SHS becomes:

$$
\begin{aligned}
\frac{d p_{0}}{d t}= & k_{p}-\frac{\lambda p_{0}^{\star}}{e^{\star}} e(t)-\lambda\left(p_{0}(t)-p_{0}^{\star}\right) \\
\frac{d p_{i}}{d t}= & \frac{\lambda p_{i-1}^{\star}}{e^{\star}} e(t)+\lambda\left(p_{i-1}(t)-p_{i-1}^{\star}\right) \\
& \quad-\frac{\lambda p_{i}^{\star}}{e^{\star}} e(t)-\lambda\left(p_{i}(t)-p_{i}^{\star}\right) \quad i=1, \ldots, n-1 \\
\frac{d p_{n}}{d t}= & \frac{\lambda p_{n-1}}{e^{\star}} e(t)+\lambda\left(p_{n-1}(t)-p_{n-1}^{\star}\right)-\gamma_{p}\left(p_{n}^{\star}\right) p_{n}^{\star} \\
& \quad-\left(\gamma_{p}\left(p_{n}^{\star}\right)+p_{n}^{\star} \gamma_{p}^{\prime}\left(p_{n}^{\star}\right)\right)\left(p_{n}(t)-p_{n}^{\star}\right) .
\end{aligned}
$$

Then, first-order moment equations readily provide the following solutions:

$$
p_{0}^{\star}=p_{1}^{\star}=\cdots=p_{n-1}^{\star}=\frac{k_{p}}{\lambda},
$$

with the steady-state solution $p_{n}^{\star}$ satisfying the following nonlinear equation

$$
\varphi\left(p_{n}^{\star}\right)=\gamma_{p}\left(p_{n}^{\star}\right) p_{n}^{\star}-k_{p}=0 .
$$

In fact, (10) admits a unique positive solution, since $\varphi\left(p_{n}^{\star}=\right.$ $0)=-k_{p}<0, \lim _{p_{n}^{\star} \mapsto+\infty} \varphi\left(p_{n}^{\star}\right)=+\infty$ and $\varphi^{\prime}\left(p_{n}^{\star}\right)>0$ for any positive $p_{n}^{\star}$ : by continuity there exists a unique positive real $p_{n}^{\star}$ that satisfies (10).

Remark 1: According to (9)-(10), it comes out that the first-order steady-state solutions $p_{0}^{\star}, p_{1}^{\star}, \ldots, p_{n}^{\star}$ do not depend of the length $n$ of the cascade. To avoid confusion, in the following, $p_{n}^{\star}$ will be shortly denoted by $\varrho$, without the possibly misleading suffix $n$.

Because of (9)-(10), the linearized system (8) simplifies into:

$$
\begin{aligned}
\frac{d p_{0}}{d t}= & k_{p}-\frac{\lambda p_{0}^{\star}}{e^{\star}} e(t)-e^{\star}\left(p_{0}(t)-p_{0}^{\star}\right) \\
\frac{d p_{i}}{d t}= & \lambda\left(p_{i-1}(t)-p_{i}(t)\right), \quad i=1, \ldots, n-1 \\
\frac{d p_{n}}{d t}= & \frac{\lambda p_{n-1}^{\star}}{e^{\star}} e(t)+\lambda\left(p_{n-1}(t)-p_{n-1}^{\star}\right)-k_{p} \\
& \quad-\left(\gamma_{p}\left(p_{n}^{\star}\right)+p_{n}^{\star} \gamma_{p}^{\prime}\left(p_{n}^{\star}\right)\right)\left(p_{n}(t)-p_{n}^{\star}\right) .
\end{aligned}
$$

It's worth noticing that intermediate average steady-states do not vary with the feedback, nor with the length of the cascade. Instead, by varying the clearance strength $\bar{\gamma}_{p}$ and the feedback parameters $\theta$ and $h$, the average steadystate solution $\varrho$ varies as described next (it can be readily verified by accounting for the fact the solution to (10) is the (unique) intersection of a fixed hyperbola, $k_{p} / \varrho$, with the Hill function, $\gamma_{p}(\varrho)$, associated to the feedback).

i) Keeping fixed the other parameters, if one increases the clearance strength $\bar{\gamma}_{p}$, then $\varrho$ reduces; instead, for $\bar{\gamma}_{p} \mapsto$ $0^{+}$, the solution $\varrho$ becomes larger and larger, and it tends to coincide with the solution without feedback for $\varrho \gg \theta$. Fig. 2 shows on a log-scale how $\varrho$ varies according to 4 different feedback sensitivities.

$$
\lim _{\bar{\gamma}_{p} \mapsto+\infty} p_{n}^{\star}=0, \quad \lim _{\bar{\gamma}_{p} \mapsto 0^{+}} p_{n}^{\star}=+\infty .
$$

ii) Keeping fixed the other parameters, if one increases the threshold $\theta$, then $\varrho$ increases; instead, for $\theta \mapsto 0^{+}$, the solution $\varrho$ tends to coincide with the one without feedback since $\varrho \gg \theta$. Fig. 3 shows on a log-scale how $\varrho$ varies according to 4 different feedback sensitivities.

$$
\lim _{\theta \mapsto+\infty} p_{n}^{\star}=+\infty, \quad \lim _{\theta \mapsto 0^{+}} p_{n}^{\star}=\frac{k_{p}}{\bar{\gamma}_{p}} .
$$

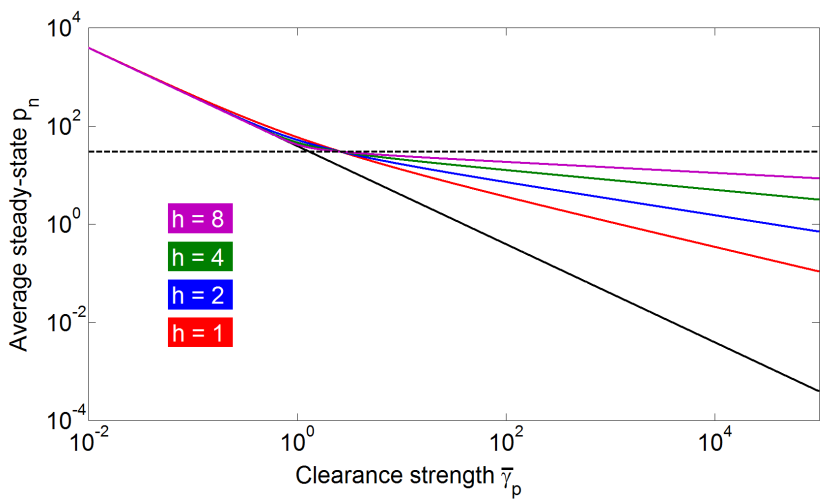

Fig. 2. Average steady-state of $p_{n}$ by varying the clearance strength $\bar{\gamma}_{p}$ The black line provides the solution without feedback that lower bounds the other feedback solutions and gets closer and closer to the feedback solutions according to a threshold $\theta \ll \varrho$. Fixed parameters $\left(k_{p}, k_{e}, \gamma_{e}, \theta, \lambda\right)$ are reported in Table II.

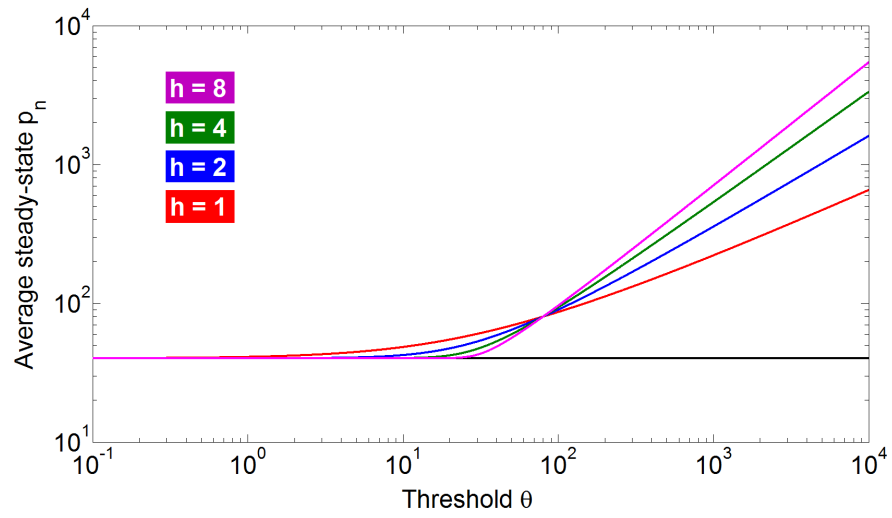

Fig. 3. Average steady-state of $p_{n}$ by varying the feedback threshold $\theta$ The black line provides the solution without feedback, which lower bounds the other feedback solutions and gets closer and closer to the feedback solutions according to a threshold $\theta \ll \varrho$. Fixed parameters $\left(k_{p}, k_{e}, \gamma_{p}\right.$, $\left.\gamma_{e}, \lambda\right)$ are reported in Table II.

In summary, with respect to the average steady-state solutions, the effect of feedback is exerted only on the final product $p_{n}^{\star}$, providing an increase (with respect to the no-feedback case) which is sharpened by increasing the feedback sensitivity $h$. The length of the cascade does not affect the average steady-state.

\section{SECOND ORDER MOMENTS}

The computation of the metabolic noise defined in (3) and (4) requires $\left\langle p_{n}^{2}\right\rangle^{\star}$. To this end, we need an algorithm to obtain the second order moments associated to (11). According to the linear fashion of (11), these moments will 
TABLE II

MOdEL PARAMETERS. MEASUREMENTS UNITS: $k_{p}, k_{e}$ [MOLECULES/TIME], $\lambda, \gamma_{e}, \bar{\gamma}_{p}$ [TIME $^{-1}$ ], $\theta$ [MOLECULES]

\begin{tabular}{|c|c|c|c|c|c|c|}
\hline Parameter & $k_{p}$ & $k_{e}$ & $\lambda$ & $\bar{\gamma}_{p}$ & $\gamma_{e}$ & $\theta$ \\
\hline Value & 40 & 10 & 10 & 1 & 1 & 30 \\
\hline
\end{tabular}

be found in closed form. To this end, denote

$$
\frac{d p_{i}}{d t}=f_{i}\left(p_{i-1}, p_{i}, e\right), \quad i=0,1, \ldots, n,
$$

the generic linear equation associated to the state $p_{i}$ in (11). Then, the following identities are derived from [19] when setting equal to zero the second order dynamics for $i, j,=$ $0,1, \ldots, n$ :

$$
\begin{aligned}
\frac{d\left\langle e p_{i}\right\rangle}{d t} & =0 \quad \Longrightarrow \quad\left\langle e f_{i}\left(p_{i-1}, p_{i}, e\right)\right\rangle+k_{e} p_{i}-\gamma_{e}\left\langle e p_{i}\right\rangle=0 \\
\frac{d\left\langle p_{i} p_{j}\right\rangle}{d t} & =0 \\
\Longrightarrow & \left\langle p_{j} f_{i}\left(p_{i-1}, p_{i}, e\right)\right\rangle+\left\langle p_{i} f_{j}\left(p_{j-1}, p_{j}, e\right)\right\rangle=0
\end{aligned}
$$

providing a square linear system of $n+1+\frac{(n+1)(n+2)}{2}$ algebraic equations with respect to the unknowns $\left\langle e p_{i}\right\rangle^{\star}$, $\left\langle p_{i} p_{j}\right\rangle^{\star}$. Below are provided the steps to compute $\left\langle p_{n}^{2}\right\rangle^{\star}$.

Step 1. From (15), the second-order moments $\left\langle e p_{i}\right\rangle^{\star}$ are found to satisfy the following closed form equation:

$$
\left\langle e p_{i}\right\rangle^{\star}=e^{\star} p_{i}^{\star}\left(1-\alpha^{i+1} \cdot C V_{e}^{2}\right)
$$

for $i=0,1, \ldots, n-1$, with

$$
\alpha=\frac{\lambda}{\lambda+\gamma_{e}},
$$

whilst $\left\langle e p_{n}\right\rangle^{\star}$ is provided by

$$
\left\langle e p_{n}\right\rangle^{\star}=e^{\star} \varrho^{\star}\left(1+\frac{\gamma_{p}(\varrho)\left(1-\alpha^{n}\right)}{\gamma_{e}+\Gamma(\varrho)} \cdot C V_{e}^{2}\right),
$$

where

$$
\Gamma(\varrho)=\gamma_{p}(\varrho)+\varrho \gamma_{p}^{\prime}(\varrho) .
$$

Remark 2: According to its definition in (18), it results $\alpha<1$. As a matter of fact, long pathways share the same insensitivity of $\left\langle e p_{n}\right\rangle^{\star}$ to $n$, since for $n$ large enough, one has $\alpha^{n} \simeq 0$ and (19) simplifies into

$$
\left\langle e p_{n}\right\rangle^{\star} \simeq e^{\star} \varrho^{\star}\left(1+\frac{\gamma_{p}(\varrho)}{\gamma_{e}+\Gamma(\varrho)} \cdot C V_{e}^{2}\right) .
$$

The length of the cascade such that (21) is a good approximation of (19) depends on the enzyme dynamics. If we increase the propensities of $e$ without varying its average steady-state $e^{\star}$ (i.e. if we proportionally increase both $k_{e}$ and $\gamma_{e}$ ), then $\lambda$ does not vary either (because it refers to the rate of modification normalized by a fixed value $e^{\star}$ ) and $\alpha$ becomes smaller. The result is that, by increasing the frequency at which the enzyme copy number varies (keeping fixed $\left.e^{\star}\right)$, then the long-cascade approximation (21) becomes accurate for smaller $n$.
Step 2. By properly exploiting the equations for $\left\langle p_{0} p_{i}\right\rangle$, $i=0,1, \ldots, n$ in (16), we find the following recursive linear system

$$
\begin{gathered}
\left\langle p_{0} p_{i+1}\right\rangle^{\star}=\frac{1}{2}\left\langle p_{0} p_{i}\right\rangle^{\star}+\frac{p_{0}^{\star} p_{i}^{\star}}{2}\left(1+\alpha^{i+2} \cdot C V_{e}^{2}\right), \\
i=0,1, \ldots, n-2, \\
\left\langle p_{0} p_{n}\right\rangle^{\star}=\frac{\lambda}{\lambda+\Gamma(\rho)}\left\langle p_{0} p_{n-1}\right\rangle^{\star}+p_{0}^{\star} \varrho\left(1-\frac{\gamma_{p}(\varrho)}{\lambda+\Gamma(\varrho)}\right) \\
-\frac{p_{0}^{\star} \varrho \gamma_{p}(\varrho)}{\lambda+\Gamma(\varrho)}\left(\frac{\lambda\left(1-\alpha^{n}\right)}{\gamma_{e}+\Gamma(\varrho)}-\alpha\right) C V_{e}^{2}
\end{gathered}
$$

with initial condition

$$
\left\langle p_{0}^{2}\right\rangle^{\star}=\left(p_{0}^{\star}\right)^{2}\left(1+\alpha \cdot C V_{e}^{2}\right) .
$$

The $n-1$ equations in (22) are recursively solved providing

$$
\left\langle p_{0} p_{n-1}\right\rangle^{\star}=p_{0}^{\star} p_{n-1}^{\star}+\frac{\alpha p_{0}^{\star} p_{n-1}^{\star}}{2^{n-1}}\left(1+\alpha \frac{1-(2 \alpha)^{n-1}}{1-2 \alpha}\right) C V_{e}^{2} \text {. }
$$

By substituting (25) into (23), we obtain $\left\langle p_{0} p_{n}\right\rangle^{\star}$ :

$$
\begin{aligned}
\left\langle p_{0} p_{n}\right\rangle^{\star}=p_{0}^{\star} \varrho+\frac{p_{0}^{\star} \varrho \gamma_{p}(\varrho)}{\lambda+\Gamma(\varrho)}\left(\frac{\alpha}{2^{n-1}}(\right. & \left.1+\alpha \frac{1-(2 \alpha)^{n-1}}{1-2 \alpha}\right) \\
& \left.-\frac{\lambda\left(1-\alpha^{n}\right)}{\gamma_{e}+\Gamma(\varrho)}+\alpha\right) C V_{e}^{2} .
\end{aligned}
$$

Similarly to what has been said in Remark 2, for $n$ large enough we have that $\left\langle p_{0} p_{n}\right\rangle^{\star}$ can be approximated by the limit

$$
\left\langle p_{0} p_{n}\right\rangle^{\star} \simeq p_{0}^{\star} \varrho\left(1-\frac{\lambda \gamma_{p}(\varrho)(\lambda-\Gamma(\varrho))}{(\lambda+\Gamma(\varrho))\left(\gamma_{e}+\Gamma(\varrho)\right)\left(\lambda+\gamma_{e}\right)} C V_{e}^{2}\right) .
$$

Step 3. We compute the steady-state solutions for $\left\langle p_{i} p_{j}\right\rangle$, with $i=1, \ldots, n, j=1, \ldots, n-1$, according to the following Lemma.

Lemma 3: Let $k=0,1, \ldots, n-1$ and define

$$
X_{k}=\left[\begin{array}{c}
\left\langle p_{1} p_{k}\right\rangle^{\star} \\
\vdots \\
\left\langle p_{n} p_{k}\right\rangle^{\star}
\end{array}\right] \in \mathbb{R}^{n} .
$$

Then, $X_{k}$ obeys the following recursive linear equation for $k=0,1, \ldots, n-2$ :

$$
X_{k+1}=A(\varrho) X_{k}+B(\varrho) U_{k},
$$

where

$$
\begin{aligned}
& A(\varrho)=\left(I_{n}-A_{2}(\varrho)\right)^{-1} A_{1}(\varrho), \\
& B(\varrho)=\left(I_{n}-A_{2}(\varrho)\right)^{-1} B_{1},
\end{aligned}
$$

with $I_{n}$ the identity matrix in $\mathbb{R}^{n \times n}$ and

$$
A_{1}(\varrho)=\operatorname{diag}\left\{\frac{1}{2}, \frac{1}{2}, \cdots, \frac{1}{2}, \frac{\lambda}{\lambda+\Gamma(\varrho)}\right\},
$$

$A_{2}(\varrho) \in \mathbb{R}^{n \times n}, B_{1} \in \mathbb{R}^{n \times 2}$ matrices with the only nontrivial elements given by

$$
\begin{aligned}
& A_{2}(i, i-1)=\frac{1}{2}, \quad i=2,3, \ldots, n-1, \\
& A_{2}(n, n-1)=\frac{\lambda}{\lambda+\Gamma(\varrho)}, \quad B_{1}(1,1)=\frac{1}{2}, \quad B_{1}(n, 2)=1 .
\end{aligned}
$$


The input $U_{k}$ in (29) is defined by

$$
U_{k}=\left[\begin{array}{c}
\left\langle p_{0} p_{k+1}\right\rangle^{\star} \\
\frac{p_{0} \varrho}{\lambda+\Gamma(\varrho)}\left(\varrho \gamma_{p}^{\prime}(\varrho)-\gamma_{p}(\varrho) \alpha^{k+1} \cdot C V_{e}^{2}\right)
\end{array}\right]
$$

Proof: The proof comes straightforwardly by computing the second order moments from (16), according to which:

$$
X_{k+1}=A_{1}(\varrho) X_{k}+A_{2}(\varrho) X_{k+1}+B_{1}(\varrho) U_{k} .
$$

Lemma 3, together with the initial conditions $X_{0}$ provided by Step 2, allows to formally write the explicit solution for $X_{n-1}$ :

$$
X_{n-1}=A(\varrho)^{n-1} X_{0}+\sum_{k=0}^{n-2} A(\varrho)^{n-k-2} B(\varrho) U_{k},
$$

from which the second order moment $\left\langle p_{n} p_{n-1}\right\rangle^{\star}$ is given by the last component of $X_{n-1}$.

According to Remark 2, for $n$ large enough, $X_{n-1}$ does not depend any more of $X_{0}$ (the first term in (35) can be neglected), since matrix $A(\varrho)$ has all positive eigenvalues and in the unit circle (indeed, the spectrum of $A$ coincides with the spectrum of $A_{1}$, as it can be readily seen by the triangular shape of the matrices). Unfortunately, no apparent easy-to-handle solutions are available for $\left\langle p_{n} p_{n-1}\right\rangle^{\star}$, even according to simplifying assumption of large $n$.

Step 4. The last step consists in the computation of $\left\langle p_{n}^{2}\right\rangle^{\star}$, coming from (16) written for $i=j=n$. Then, after computations:

$$
\begin{aligned}
\left\langle p_{n}^{2}\right\rangle^{\star}=\varrho^{2} \cdot\left(1-\frac{\gamma_{p}(\varrho)}{\Gamma(\varrho)}\right)+\frac{\lambda}{\Gamma(\varrho)}\langle & \left.p_{n} p_{n-1}\right\rangle^{\star} \\
& +\frac{k_{p}^{2}\left(1-\alpha^{n}\right)}{\Gamma(\varrho)\left(\gamma_{e}+\Gamma(\varrho)\right)} \cdot C V_{e}^{2},
\end{aligned}
$$

with $\left\langle p_{n} p_{n-1}\right\rangle^{\star}$ achieved in Step 3. $C V_{p, n}^{2}$ is straightforwardly computed according to (3).

Remark 4: In summary, with respect to the way the noise scales with the length of the metabolic pathway, analytical solutions for the second-order moments highlight the fact that the influence of $n$ is related to the value of the proper fraction $\alpha$, since $n$ appears in the moments computations in the form of $\alpha^{n}$. The values of $C V_{p, n}^{2}$ converge to a limit point when $n \mapsto+\infty$, according to a rate given by $\alpha$ : the smaller is $\alpha$ (i.e. the closer to zero), the faster is the rate. As previously stated in Remark $2, \alpha$ is related to the enzyme dynamics.

The way the feedback influences the noise propagation will be discussed by simulations in the next section.

\section{NUMERICAL SIMULATIONS}

Numerical simulations have been carried out in order to evaluate how the feedback scales with noise propagation. Figs. 4 and 5 report the standard deviation according to different values of the clearance strength $\bar{\gamma}_{p}$ and of the threshold $\theta$. Other parameters can be found in Table II. Continuous lines refer to the shortest cascade with only $p_{0}$, $p_{1}, p_{2}$, whilst dotted lines refer to the case of $n=12$. Parameters are set providing $\alpha=10 / 11$ closer to 1 than to 0 , thus allowing the length $n$ to play a nontrivial role. By comparing Figs. 4-5 with Figs. 2-3 it is apparent that for values of $\bar{\gamma}_{p}$ (and of $\theta$ ) small enough to provide an average steady-state $\varrho \gg \theta$, the feedback does not exert a real action (because the Hill function in (2) can be confused with $\bar{\gamma}_{p}$ ) and the standard deviation without the feedback (black line) is not modified by the feedback (colored lines). Instead, by increasing $\bar{\gamma}_{p}$ in Fig. 4 (and $\theta$ in Fig. 5), the feedback exerts its action and both figures show an overall increase in $\sigma$, with the curves showing non-monotonicity for the feedback sensitivity $h>1$. However, this fact does not provide a corresponding increase in the metabolic noise (quantified by the coefficient of variation defined in (3)) because the feedback leads also to an increment of the steady-state $\varrho$. This fact can be appreciated by the plot of the feedback relative noise (4), where it is apparent (in Figs. 6-7) how the feedback strongly reduces $\eta_{n}^{2}$ according to a monotonic decrease, which becomes sharper by increasing the feedback sensitivity $h$.

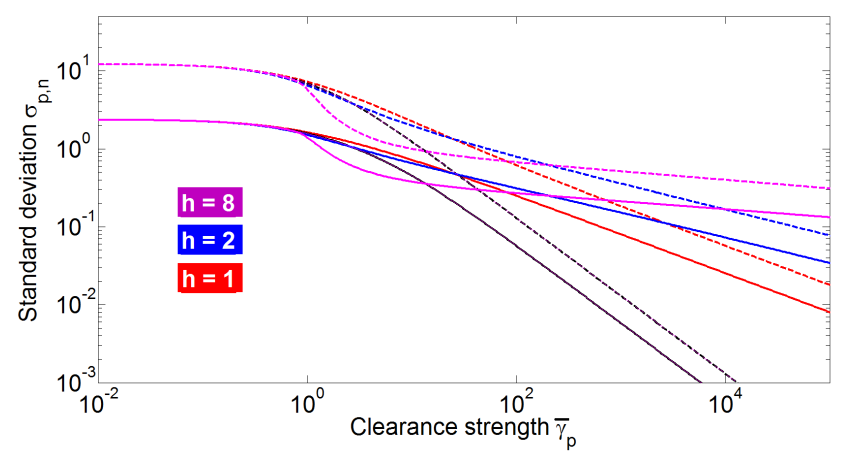

Fig. 4. Standard deviation of $p_{n}$ by varying the clearance strength $\bar{\gamma}_{p}$ The black line provides the solution without feedback. Fixed parameters $\left(k_{p}, k_{e}, \gamma_{e}, \theta, \lambda\right)$ are reported in Table II. Continuous lines refer to $n=2$; dotted lines refer to $n=12$.

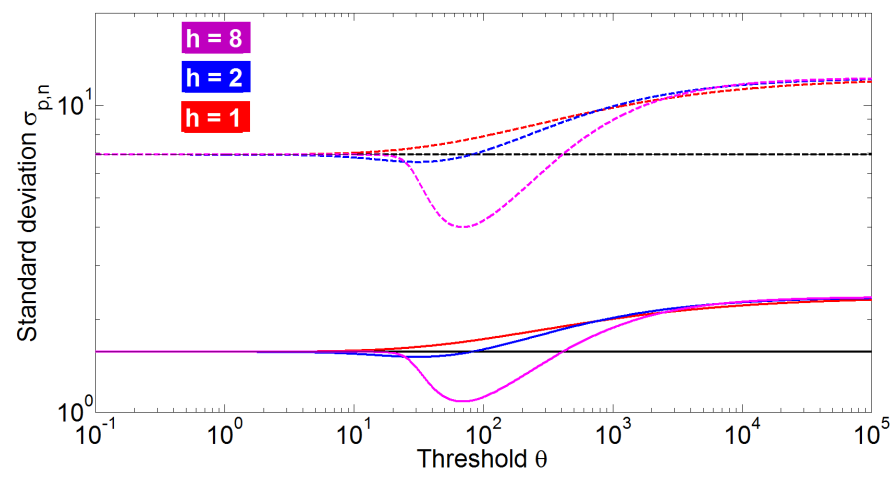

Fig. 5. Standard deviation of $p_{n}$ by varying the feedback threshold $\theta$. The black line provides the solution without feedback. Fixed parameters $\left(k_{p}\right.$, $\left.k_{e}, \gamma_{p}, \gamma_{e}, \lambda\right)$ are reported in Table II. Continuous lines refer to $n=2$; dotted lines refer to $n=12$.

Finally, Figs. 8-9 report numerical results on the steadystate $\varrho$ and $\left\langle p_{n}^{2}\right\rangle^{\star}$ computed by means of the Gillespie Stochastic Simulation Algorithm (SSA) [22], properly modified to take into account the SHS framework. Stochastic 


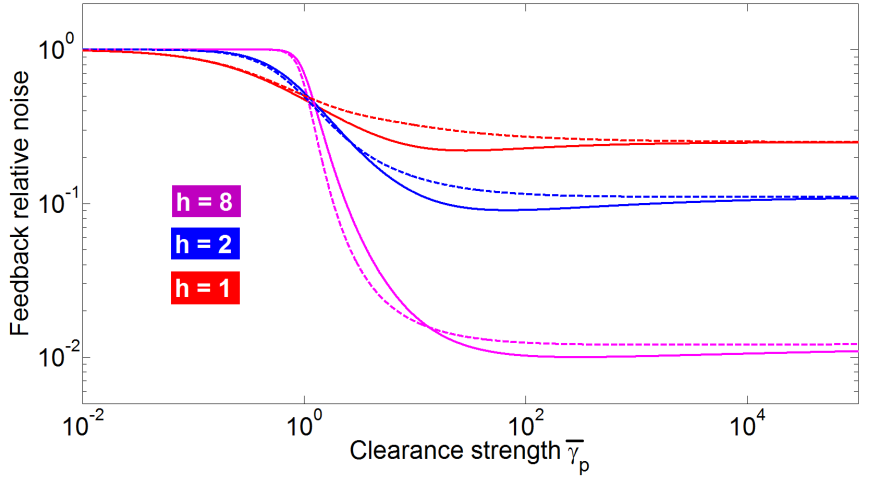

Fig. 6. Feedback relative noise versus the clearance strength $\bar{\gamma}_{p}$ for different values of the feedback sensitivity. The Hill coefficient has been fixed to $h=4$. Fixed parameters $\left(k_{p}, k_{e}, \gamma_{p}, \gamma_{e}, \theta\right)$ are reported in Table II. Continuous lines refer to $n=2$; dotted lines refer to $n=12$.

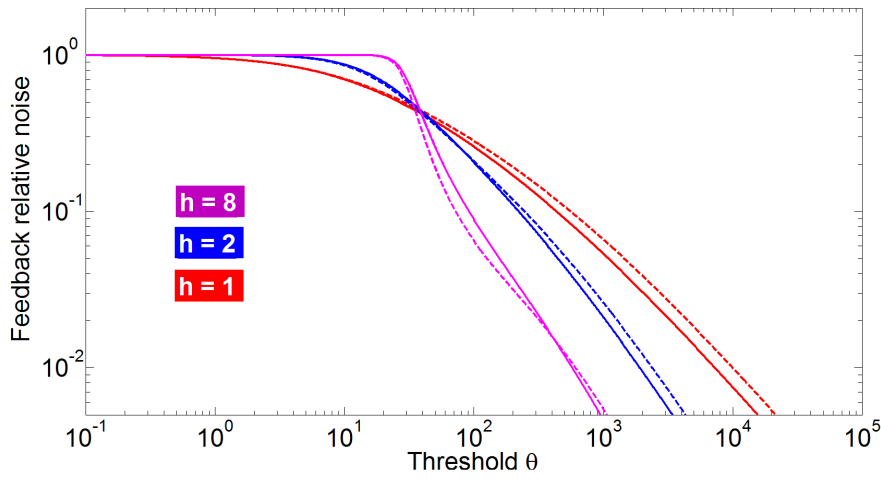

Fig. 7. Feedback relative noise versus the threshold $\theta$ for different values of the feedback sensitivity. The Hill coefficient has been fixed to $h=4$. Fixed parameters $\left(k_{p}, k_{e}, \gamma_{p}, \gamma_{e}, \theta\right)$ are reported in Table II. Continuous lines refer to $n=2$; dotted lines refer to $n=12$.

simulations are carried out without the linearization and are very close to the linear SHS approximation: they apparently show that the linear approximation adopted to achieve closed moment equations with SHS is a reasonable tradeoff to lighten the computational burden, still keeping meaningful results.

\section{CONCLUSIONS}

In this note, a study on noise propagation in a class of metabolic networks has been carried out. The biological system under investigation is a cascade of metabolic reactions, eventually leading to the formation of a final product by means of intermediate substrate transformations. The formalization adopted is a Stochastic Hybrid System (linearized in order to achieve closed moment equations), with the only noise source provided by the production/clearance processes associated to the copy number variations of the enzyme $e$. Noise propagation has been investigated with respect to the length of the cascade and with respect to the action exerted in feedback on the final product clearance rate by the product accumulation itself. In addition to showing the unmistakable role of the feedback in noise reduction (a positive feedback,

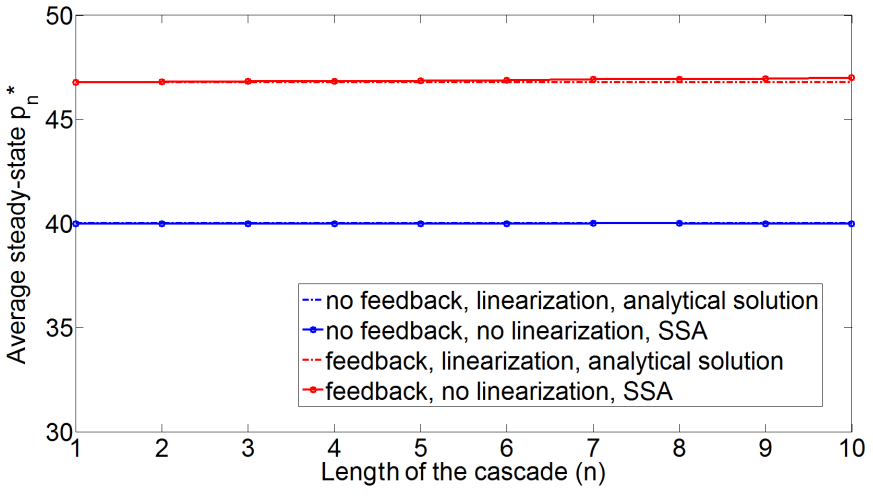

Fig. 8. Average steady-state $\varrho$ versus the length of the cascade $n$. Comparison between linearized SHS results and non-linearized Gillespie numerical simulation. All parameters (except $n$ ) are fixed to the values of Table II.

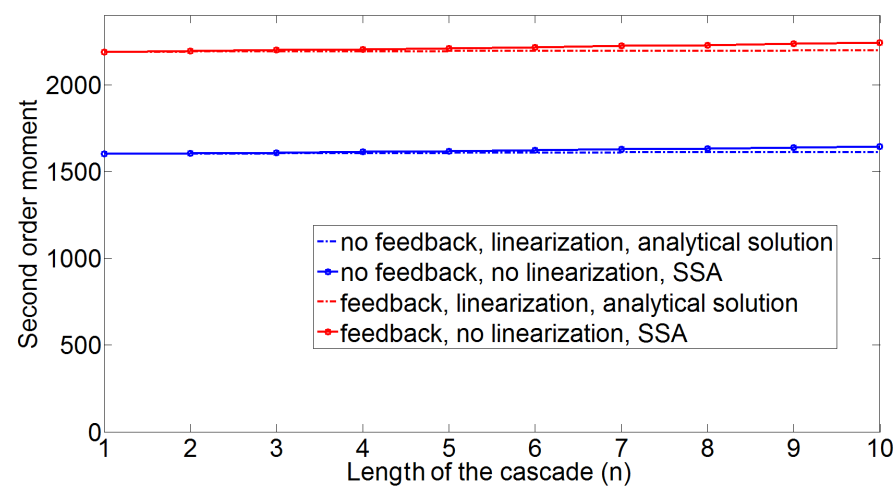

Fig. 9. Second order moments $\left\langle p_{n}^{2}\right\rangle^{\star}$ versus the length of the cascade $n$. Comparison between linearized SHS results and non-linearized Gillespie numerical simulation. All parameters (except $n$ ) are fixed to the values of Table II.

in this case), analytical results allow to investigate the impact of different model parameters on noise regulation where, in particular, an interesting result correlates the impact of the length $n$ of the cascade to the enzyme dynamics.

\section{ACKNOWLEDGEMENTS}

PP is supported by the MIUR grant SysBioNet Italian Roadmap for ESFRI Research Infrastructures, SYSBIO Centre of Systems Biology, Milan and Rome, Italy.

AS is supported by the National Science Foundation Grant DMS-1312926.

\section{REFERENCES}

[1] D.H. Calhoun, G.W. Hatfield, Autoregulation: a role for a biosynthetic enzyme in the control of gene expression, Proceedings of the National Academy of Sciences 70.10 (1973): 2757-2761.

[2] G. Stephanopoulos, A. Aristidou, J. Nielsen, Metabolic Engineering: Principles and Methodologies, Academic Press, San Diego, CA, 1998

[3] A. Zaslaver, A. Mayo, R. Rosenberg, P. Bashkin, H. Sberro, M. Tsalyuk, M. Surette, U. Alon, Just-in-time transcription program in metabolic pathways, Nat. Genet. 36, 486-491, 2004.

[4] U. Alon, An Introduction to Systems Biology: Design Principles of Biological Circuits, Chapman and Hall/CRC, 2006. 
[5] A. Singh, J.P. Hespanha, Optimal feedback strength for noise suppression in autoregulatory gene networks, Biophysical Journal 96, 40134023, 2009.

[6] W.J. Holtz, J.D. Keasling, Engineering static and dynamic control of synthetic pathways, Cell, 140, 19-23, 2010.

[7] Oyarzun, D.A, Lugagne, J.-B., Stan, G.-B.V.: Noise propagation in synthetic gene circuits for metabolic control, ACS Synthetic Biology, 2014.

[8] A. Borri, P. Palumbo, A. Singh, Impact of negative feedback in metabolic noise propagation, IET Syst. Biol., 1-8, 2016.

[9] A. Borri, P. Palumbo, A. Singh, Metabolic noise reduction for enzymatic reactions: the role of a negative feedback, Proceedings of the 54th IEEE Conference on Decision and Control (CDC 2015), Osaka, Japan, pp. 2537-2542, 2015.

[10] A. Borri, P. Palumbo, A. Singh, Noise reduction for enzymatic reactions: a case study for stochastic product clearance, Proceedings of the 55th IEEE Conference on Decision and Control (CDC 2016), Las Vegas, USA, pp. 5851-5856, 2016.

[11] E.D. Sontag, Some new directions in control theory inspired by systems biology, Syst. Biol. 1(1) (2004): 9-18.

[12] D.E. Cameron et al, A brief history of synthetic biology, Nat. Rev. Microbiol., 12, 381-390, 2014.

[13] D. Del Vecchio, and E. D. Sontag, Synthetic biology: A systems engineering perspective, Control Theory and Systems Biology, 101124, 2009.

[14] D. Del Vecchio, A. J. Ninfa, and E. D. Sontag, Modular cell biology: retroactivity and insulation, Molecular systems biology, 4.1, 161, 2008.

[15] D. Del Vecchio, Modularity, context-dependence, and insulation in engineering biological circuits, Trends in Biotechnology, 33(2), 111119, 2015.

[16] D. Del Vecchio, A.J. Dy, Y. Qian, Control theory meets synthetic biology, J. R. Soc. Interface 13, 13: 20160380. http://dx.doi.org/10.1098/rsif.2016.0380

[17] D.J. Kiviet, P. Nghe, N. Walker, S. Boulineau, V. Sunderlikova, S.J. Tans, Stochasticity of metabolism and growth at the single-cell level. Nature, 514, 376-379, 2014.

[18] A. Singh and J. P. Hespanha, Scaling of stochasticity in gene cascades, Proceeding of the American Control Conference (ACC 2008), Seattle, WA, pp. 2780-2785, 2008.

[19] J.P. Hespanha, A. Singh, Stochastic models for chemically reacting systems using polynomial stochastic hybrid systems, Int. J. of Robust and Nonlinear Control, 15, 669-689, 2005.

[20] A. Singh, J.P. Hespanha, Approximate moment dynamics for chemically reacting systems, IEEE Transactions on Automatic Control. 56, 414-418, 2011.

[21] E. D. Sontag and A. Singh, Exact Moment Dynamics for Feedforward Nonlinear Chemical Reaction Networks, in IEEE Life Sciences Letters, vol. 1, no. 2, pp. 26-29, Aug. 2015.

[22] D. T. Gillespie, Exact Stochastic Simulation of Coupled Chemical Reactions, The Journal of Physical Chemistry 81(25), 23402361, 1977. 